\title{
Demonstration of Photon-Echo Rephasing of Spontaneous Emission
}

\author{
Sarah E. Beavan, ${ }^{*}$ Morgan P. Hedges, and Matthew J. Sellars \\ Centre for Quantum Computation and Communication Technology, RSPE, Australian National University, \\ Canberra, ACT 0200, Australia
}

(Received 4 May 2012; published 29 August 2012)

\begin{abstract}
In this paper we report the first demonstration of "rephased amplified spontaneous emission" (RASE) with photon-counting detection. This protocol provides an all-in-one photon-pair source and quantummemory that has applications as a quantum repeater node. The RASE protocol is temporally multimode, and in this demonstration the photon echo was generated in a way that is spatially multimode and includes intermediate storage between two potentially long-lived spin states. A correlation between spontaneous emission and its photon echo was observed, using an ensemble of $\operatorname{Pr}^{3+}$ ions doped into a $\mathrm{Y}_{2} \mathrm{SiO}_{5}$ crystal. Alterations that would allow for the measurement of nonclassical correlations are identified. These should generally apply for future experiments in rare-earth ion crystals, which are promising systems for implementing highly-multiplexed quantum repeater operations.
\end{abstract}

PACS numbers: 42.50.Ex, 03.67.-a, 32.80.Qk, 78.47.jf

Performing quantum-encrypted communications over long distances will require a quantum repeater. Such a repeater is likely to involve on-demand conversion between entangled photonic states and atomic based memories [1,2]. A significant conceptual advance came with the realization that an ensemble of atoms could be used to achieve a quantum repeater [3]. Among other advantages, this avoids the need to strongly couple a single atom to a given field mode. Sharing excitation across an ensemble of atoms guarantees a well-defined spatial mode for the photonic states, given by the direction of constructive interference of emission from all the individual atoms. Specifically, the seminal "DLCZ" proposal is based on spontaneous Raman transitions in an ensemble of $\Lambda$-type energy level systems [3].

There has been significant experimental efforts towards a quantum repeater in the DLCZ framework since its inception a decade ago (for the latest results, see, for example, Refs. [4,5]), however a scalable quantum repeater is yet to be realized. It has become evident that efficient multiplexing will be required in order to implement a practical quantum repeater which actually outperforms the direct transmission of light states [2]. As with other ensemble techniques, the DLCZ protocol can be spatially multimode, however only a single temporal mode can be stored.

Recently, Ledingham et al. described an alternative protocol for generating pairs of photons with intermediate quantum storage [6]. Similarly to the DLCZ proposal, this uses a collective ensemble state generated via the measurement of spontaneous emission. In contrast to the DLCZ method, this proposal applies photon-echo techniques to read out the ensemble state, and thus is inherently temporally multimode. This protocol is referred to as "rephased amplified spontaneous emission", or RASE [7].

In this Letter we report an experimental demonstration of the RASE protocol. The system used for this demonstration is a rare-earth ion doped crystal (REIC). These solid-state systems have received much attention in recent years for quantum information processing applications, particularly in the context of quantum memories. This is in part due to the fact that it is possible to have long coherence times at both optical $(\sim 100 \mu \mathrm{s})$ and $\mathrm{RF}$ frequencies $(\sim$ seconds $[10,11]$, and potentially longer still [12]). Further, being a solid means there is no spatial diffusion. Therefore collective atomic states remain coherent, allowing a large number of spatial modes to be accessed within a single ensemble.

Although there have been demonstrations of on-demand quantum memories using REIC ([13,14]), and there is potential for long coherence times, a repeater architecture is yet to be realized. The main difficulty is in combining the narrow-bandwidth REIC memories (limited to tens-of$\mathrm{MHz}$ due to the spin-state splittings) with a compatible source of entangled photon pairs.

One approach is to integrate these existing memory protocols with heavily-filtered broad-bandwidth photon pairs produced using spontaneous parametric downconversion. There have been recent demonstrations towards this goal, where entanglement was generated between a telecom wavelength photon and a collective ensemble state. This was demonstrated in a $120 \mathrm{MHz}$ wide atomic frequency comb (AFC) in a bulk crystal [15], as well as a $5 \mathrm{GHz}$ wide AFC in a waveguide [16]. However further bandwidth reduction is necessary before the atomic states can be transferred from an optical- to a spintransition. This transfer is required for the AFC to constitute an efficient on-demand memory.

Methods such as RASE and DLCZ represent an alternative means to achieve a quantum repeater in REIC, where the photons are produced by the same atoms that store the excitation in a single-step process. In this Letter we investigate the RASE proposal, which directly exploits the long coherence times available on the optical 
transitions in REICs to store multiple temporal modes, while utilizing the spin transitions for long-term coherent storage.

The basic RASE protocol is performed using an ensemble of inhomogeneously broadened "two-level" atoms, where the population is initially in the ground state. A $\pi$ pulse is applied to invert the ensemble. Following this, spontaneous emission is detected in the far field [17]. Since there is no information as to which atom(s) spontaneously decayed, each detected emission event heralds entanglement amongst the atoms in the ensemble. Because of the inhomogeneous broadening, this particular ensemble state will dephase. However, the inhomogeneity can be rephased through the application of a $\pi$ pulse, similarly to a photon-echo [18]. Following the rephasing $\pi$ pulse, the ensemble evolves and radiates a time-reversed copy of the initial spontaneous emission sequence, in a well-defined spatial mode.

This RASE demonstration was enabled by applying a novel photon-echo pulse sequence utilizing four atomic levels. The characterization of this four-level echo (4LE) sequence was reported in reference [19]. The 4LE allows us to distinguish the signal (at the single-photon level) from coherent emission induced by the inhomogeneous rephasing pulses ( $\sim \mathrm{mW}$ intensity). Furthermore, this sequence includes transferral of the optical coherence to a spin-transition, where coherence can potentially be stored for seconds.

The application of this pulse sequence to rephase spontaneous emission is depicted in frequency and temporal domains in Figs. 1(b) and 1(c) respectively. Essentially, the 4LE sequence rephases coherence between two levels (here $|3\rangle \leftrightarrow|4\rangle$ ) while transferring it to a completely different transition $(|2\rangle \leftrightarrow|5\rangle)$. This is achieved through the sequential application of two $\pi$ pulses, driving transitions $|2\rangle \leftrightarrow|4\rangle$ and $|3\rangle \leftrightarrow|5\rangle$. As a result, the free-induction decay (FID) emission following both the bright rephasing pulses occurs at a different frequency to both the ASE and RASE fields. Moreover, due to the flexible phase-matching condition, the FID can also be spatially resolved from the signal [19]. Here we use a counterpropagating phase-matching arrangement; where $\mathbf{k}_{\pi_{1}}+\mathbf{k}_{\pi_{2}}=0$ and $\mathbf{k}_{\mathrm{RASE}}+\mathbf{k}_{\mathrm{ASE}}=0$. With this geometry, RASE with the 4LE for rephasing is intrinsically spatially multimode.

The sample used in this experiment was a $20 \times 4 \times$ $4 \mathrm{~mm} 0.005 \% \mathrm{Pr}^{3+}: \mathrm{Y}_{2} \mathrm{SiO}_{5}$ crystal, cooled to $\sim 2 \mathrm{~K}$ in a liquid helium bath cryostat. This material is a common choice for REIC demonstrations ([10,13,14]), because relatively long coherence times can be attained on transitions with reasonable oscillator strengths. The energy level structure is depicted in Fig. 1(b). The excited state lifetime $\left(T_{1}\right)$ is roughly $160 \mu \mathrm{s}$, the coherence time $\left(T_{2}\right)$ is $150 \mu \mathrm{s}$ [20], and $T_{1}$ between ground state hyperfine levels is about $100 \mathrm{~s}$. All optical transitions are weakly allowed. The various transition strengths are reported in Ref. [21]. (a)

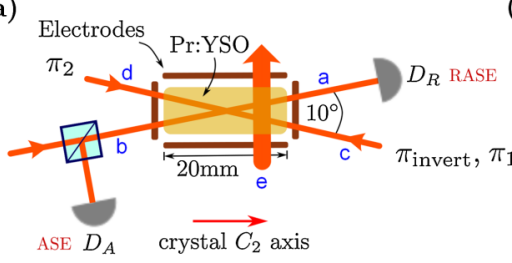

(b)

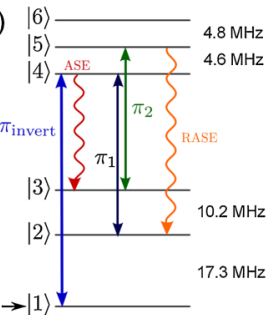

(c)

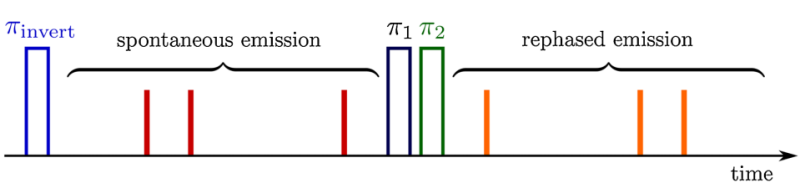

FIG. 1 (color online). Overview of key experiment parameters for demonstrating RASE with the 4LE. (a) Counterpropagating beam geometry, with path labels $a$ through $e$ defined. The ASE and RASE modes are detected using APDs labeled $D_{A}$ and $D_{R}$ respectively. There is a 50:50 beam splitter before the ASE detector to allow for spectral hole burning along path $b$. (b) The ${ }^{3} H_{4}(1) \rightarrow{ }^{1} D_{2}(1)$ site 1 transition in $\operatorname{Pr}^{3+}: \mathrm{Y}_{2} \mathrm{SiO}_{5}$, which is at $606 \mathrm{~nm}$ [20]. The ground and excited state is split into three doubly degenerate spin states with separations $\sim 10 \mathrm{MHz}$. For the RASE experiment a subensemble of ions is selected using spectral hole burning, and prepared to be initially in state $|1\rangle$. The frequencies used to apply the 4LE are marked. (c) Temporal schematic of a single shot of the RASE experiment. In this example, there are three correlated pairs of photonic states produced.

The beam geometry is depicted in Fig. 1(a). Acoustooptic modulators are used in double-pass configurations to gate the dye laser (sub-kHz linewidth), and also shift the frequency in the $\mathrm{MHz}$ range. The counterpropagating $\pi$ pulses are applied along path $c-d$, and the ASE and RASE fields are detected using avalanche photodiodes (APDs) along path $a-b$. These paths are separated by $10^{\circ}$, such that they overlap within the sample in a region $\sim 2 \mathrm{~mm}$ long. This overlap region defines the ensemble of ions for the experiment.

The ${ }^{3} H_{4} \rightarrow{ }^{1} D_{2}$ transition in this crystal is inhomogeneously broadened to $\sim 5 \mathrm{GHz}$, which is much wider than the hyperfine splittings. However, prior to performing a single shot of the RASE experiment, a subgroup of ions with just $\sim 200 \mathrm{kHz}$ inhomogeneity is selected out and initialized using spectral hole-burning techniques [22]. The spectral hole-burning process is similar to that described in Ref. [21].

Since the APDs are not sensitive to frequency, all of the necessary spectral filtering of the ASE and RASE modes must be performed prior to detection. Most of the excited state population $(\sim 97 \%)$ decays to the ground state via intermediate crystal field levels. This emission occurs upwards of $610 \mathrm{~nm}$, and is attenuated with a bandpass interference filter placed before each APD detector. A more difficult filtering requirement is to distinguish the ASE and RASE signals from the spontaneous emission to the other ground states, occurring just $\mathrm{MHz}$ away. Here we achieve 
this, for the RASE detection mode, using spectral holeburning in a second region of the sample. This is enacted by burning antiholes at the predicted noise frequencies along beam path $e$ [Fig. 1(a)] [22]. This improves the signal to noise of the RASE detection by absorbing the spontaneous emission from any unwanted population in the excited states $|4\rangle$ or $|5\rangle$. The resulting attenuation was characterized using heterodyne detection, and was found to be roughly $26 \mathrm{~dB}$.

The 4LE coherence time was measured to be $80 \mu \mathrm{s}$. This is half of the coherence time measured for a simple two-level Hahn echo, which is attributed to a component of inhomogeneity amongst the spin levels that is not rephased by this sequence [19].

A single shot of the RASE experiment is performed after selecting out the experiment ensemble and hole burning the RASE filter (which takes approximately $200 \mathrm{~ms}$ ). The temporal sequence is outlined in Fig. 1(c). The first pulse applied is the inversion $\pi$ pulse along path $c$. This pulse propagates through the entire $20 \mathrm{~mm}$ length of an optically thick medium $(\sim 80 \mathrm{~dB} / \mathrm{cm}$ absorption). However, the experiment ensemble lies in the middle $2 \mathrm{~mm}$ section. In order to avoid significant superradiance due to the large gain along path $c-d$, an electric field gradient is applied during the propagation of the $\pi_{\text {invert }}$ pulse to broaden the gain feature. This is achieved using a quadrupole electrode arrangement similar to that used in Ref. [13].

Having determined the properties of the inversion feature, the filter transmission, and the 4LE coherence time, the RASE experiment itself was performed. The histogram of counts is shown in Fig. 2. Evidence of RASE can be seen in the signal measured by $D_{R}$, which exhibits a region of high count rates for the $20 \mu$ s period following the rephasing pulses. This is comparable in width to the ASE emission window. Moreover, if the second $\pi$ pulse is not applied, and therefore the ensemble state is not rephased,

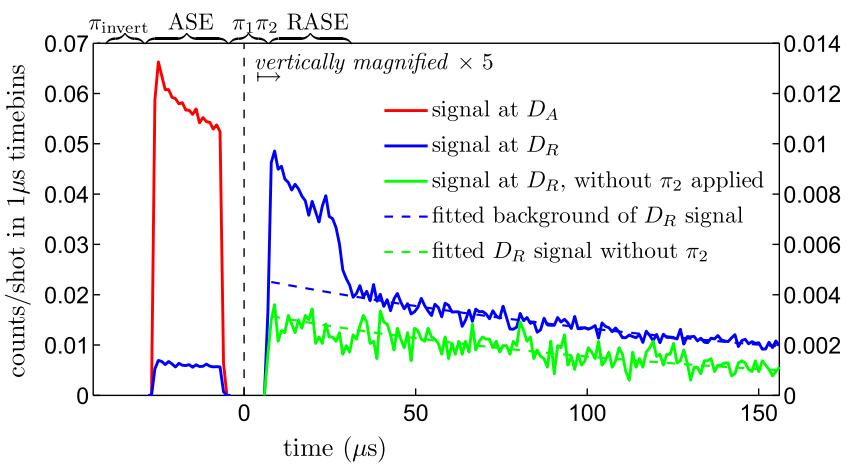

FIG. 2 (color online). Histogram of photon counts recorded by the detectors in the RASE experiment, averaged over $10^{5}$ shots. Both detectors were gated on during the ASE and RASE temporal periods, however the $D_{A}$ histogram (red trace) is only plotted before the rephasing $\pi$ pulses for clarity. The detectors are gated on for $20 \mu \mathrm{s}$ following the inversion pulse. The zero on the time axis represents the center point of the rephasing pulses. this peak disappears. The rephasing efficiency was measured to be $16 \% \pm 3 \%$, with a signal to noise ratio of the RASE mode of 0.9 .

The histogram (Fig. 2) suggests a correlation between ASE and RASE intensity averaged across all shots. A more thorough test is to calculate the degree of correlation on a single shot basis. The correlation between the photon streams is quantified using the second-order crosscorrelation function, $g_{A, R}^{(2)}(\tau)$, defined as [23];

$$
g_{A, R}^{(2)}(\tau)=\frac{1}{N_{T}} \sum_{t=T_{0}}^{T_{\max }} \frac{\left\langle n_{A}(t) n_{R}(t+\tau)\right\rangle}{\left\langle n_{A}(t)\right\rangle\left\langle n_{R}(t+\tau)\right\rangle},
$$

where \langle\rangle denotes the average over all shots, $n_{A}(t)$ is a number of counts detected by $D_{A}$ at time $t$ before the rephasing pulses, and $n_{R}(t+\tau)$ is the counts detected by $D_{R}$ at time $t+\tau$ after the rephasing pulses. The average is also taken in a single shot across a number of time bins, spanning from $T_{0}$ to $T_{\max }$, with the normalization factor $N_{T}$ representing the number of time bins spanned. For completely independent ASE and RASE counts, the numerator of Eq. (1) factorizes, and $g_{A, R}^{(2)}(\tau)=1$. If there is a correlation, then $g_{A, R}^{(2)}>1$, and for a photon-echo process this will be maximal at $\tau=0$.

The second-order cross-correlation function is plotted in Fig. 3. There is clearly a peak, which confirms the prediction that spontaneous emission can indeed be rephased. The data is fitted with a Gaussian of amplitude $1.21 \pm 0.03$. The correlation was also calculated between the ASE and RASE modes, but using data from different shots. The average across 10 neighboring shots, also shown in Fig. 3, shows no evidence of correlations due to slowly varying external factors, such as a fluctuating laser power.

The correlation-peak width is $4.4 \pm 0.8 \mu \mathrm{s}$, which is consistent with the expected $150 \mathrm{kHz}$ bandwidth of the

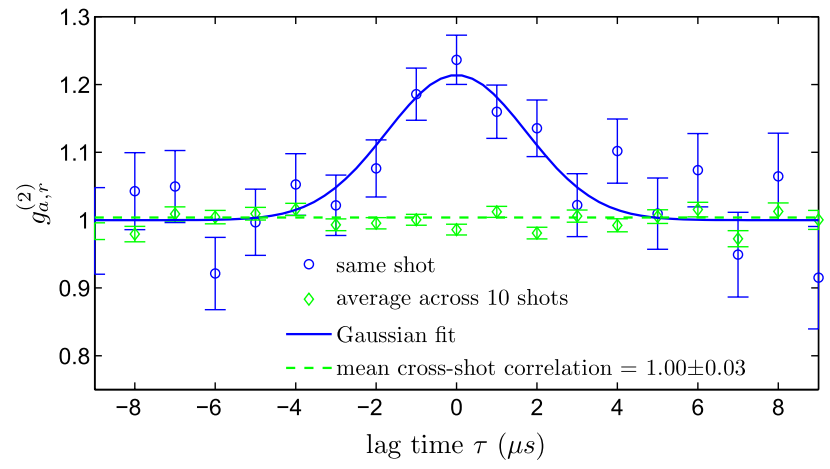

FIG. 3 (color online). The correlation between ASE and RASE temporal modes is quantified using the second-order correlation function [see Eq. (1)] with $1 \mu \mathrm{s}$ time bins, and $T_{\max }-T_{0}=$ $14 \mu \mathrm{s}$. The data is fitted with a Gaussian peak of amplitude $1.21 \pm 0.03$ and width $t_{c}=4.4 \pm 0.8 \mu \mathrm{s}$. The green diamonds represent the cross-correlation average across 10 adjacent shots. This averages to $1.00 \pm 0.03$. 
inversion $\pi$-pulse. The average number of ASE photons emitted in a single temporal-mode $(4.4 \mu \mathrm{s})$, determined using the count histograms and the measured transmission efficiency along the detector paths, is $\left\langle n_{A}\right\rangle=3 \pm 0.3$. Given the recording period of $20 \mu \mathrm{s}$, the time-bandwidth product of the RASE experiment performed here is $\sim 5$.

For this protocol to be used in quantum communication, it is necessary that the degree of correlation between the photon pairs is stronger than can be obtained with classical signals; i.e., that they are entangled. An initial test for a nonclassical nature in terms of these photon-counting measurements can be derived from the Cauchy-Schwarz inequality. A classical description of the field requires that the following relationship between cross- and autocorrelations holds: $g_{A, R}^{(2)} \leq \sqrt{g_{A, A}^{(2)} \times g_{R, R}^{(2)}}$ [23]. For any singlefrequency incoherent radiation source, the autocorrelation at $\tau=0$ is 2 ; this effect is referred to as "photon bunching." However as multiple modes of such fields are mixed together, this bunching effect is reduced and the autocorrelation approaches 1 . The values measured in this experiment for the autocorrelations were $g_{A, A}^{(2)}=1.46 \pm 0.01$ and $g_{R, R}^{(2)}=1.66 \pm 0.09$ [22]. This indicates that there is background noise present in each field, and, in particular, for the ASE mode in which there is no hole burnt frequency filter.

Given that the measured values of $g_{A, A}^{(2)}$ and $g_{R, R}^{(2)}$ are each larger than the cross-correlation, the measured degree of correlation between ASE and RASE is clearly in the classical realm. This indicates that the degree of added incoherent noise present masks the nonclassical nature of the ASE and RASE intensity correlations.

Some spectral properties of the background noise can be inferred using the histogram where the second rephasing pulse $\left(\pi_{2}\right)$ was omitted (see Fig. 2). The counts detected without $\pi_{2}$ are due to spontaneous emission from residual population in excited state |4〉. This emission is all distinguishable in frequency from the RASE signal. Modeling suggests that improving the filtering so as to significantly reduce this noise component will allow for a nonclassical correlation measurement.

A more fundamental problem with implementing RASE in REIC is that there is a component of noise that is indistinguishable from the RASE signal. This is seen by comparing the $D_{R}$ signals in Fig. 2 for time $>25 \mu \mathrm{s}$ with and without $\pi_{2}$ applied. This added noise emission is mostly due to ions that decayed to ground state $|3\rangle$ indirectly, i.e., via the intermediate crystal field levels. Upon excitation to state $|5\rangle$ this group of ions can radiate in a mode overlapping with the RASE signal.

This problem is associated with the low quantum efficiency of the ${ }^{3} H_{4} \rightarrow{ }^{1} D_{2}$ transition. To reduce this noise, we propose implementing RASE in a sample placed inside a resonant cavity. By doing so we could achieve an identical level of gain, with fewer ions initially excited, and thus reduce the number of ions that can radiate uncorrelated emission. This noise emission should reduce in intensity linearly with the cavity finesse. Therefore a moderate finesse of $\sim 100$ should suffice to remove $99 \%$ of this noise.

The possibility for measuring a strong violation of the Cauchy-Schwarz inequality can also be enhanced by reducing the signal itself. In the absence of noise, the ratio of the cross to the autocorrelation functions can be written in terms of the mean ASE photon number per mode $\langle n\rangle$ as

$$
R=\frac{g_{A, R}^{(2)}}{\sqrt{g_{A, A}^{(2)} \times g_{R, R}^{(2)}}}=1+\frac{1}{2\langle n\rangle} .
$$

In practice, $\langle n\rangle$ is reduced by inverting fewer ions initially and thus reducing the gain. With $\langle n\rangle \ll 1$, then $R \gg 1$, which is highly nonclassical. This low photon number regime is relevant for applications as a single-photon source, or quantum repeater node. However, nonclassical states of larger mean photon number may also be generated with the RASE protocol, in which case a different metric (e.g., dB of squeezing) may be more appropriate.

In conclusion, we have verified that spontaneous emission is rephased by a photon-echo sequence. The degree of correlation measured was insufficient to verify nonclassical behavior due to added noise in the detection mode. The majority of this noise can be negated with improved frequency discrimination. A spectrally-indistinguishable component of the noise has also been identified, and the use of a cavity is proposed to eliminate this effect.

Nonclassical correlations between RASE and ASE modes have been demonstrated recently in a non-holeburning REIC (with a more favorable branching ratio) using continuous variable detection [24]. This result confirms the expected behavior when the noise sources identified in this Letter may be neglected. However, holeburning materials like $\mathrm{Pr}^{3+}: \mathrm{Y}_{2} \mathrm{SiO}_{5}$ are used for most REIC quantum information demonstrations due to their flexibility and potential for long-term spin shelving. Such materials will most likely be required for practical devices such as repeaters, both for the long-term storage and compatibility with other quantum-memory protocols. The current Letter demonstrates a method by which RASE may be implemented in such high coherence hole-burning materials. Further, it shows that single-photon detection, as required for current repeater proposals, is practical if appropriate filtering steps are taken.

The RASE protocol in REIC systems is a promising methodology for the generation of entangled light fields with built-in on-demand quantum memory. The RASE protocol serves as an improvement over DLCZ for rareearth ion based repeaters, allowing temporal as well as spatial multiplexing. Further, it is compatible with recent advances in REIC quantum memory such that a broad range of quantum processing applications can now be considered. 
The authors acknowledge the support of DSTO, and the Australian Research Council Centre of Excellence for Quantum Computation and Communication Technology (Project No. CE110001027).

*Sarah.Beavan@anu.edu.au

[1] H.-J. Briegel, W. Dür, J. I. Cirac, and P. Zoller, Phys. Rev. Lett. 81, 5932 (1998).

[2] N. Sangouard, C. Simon, H. de Riedmatten, and N. Gisin, Rev. Mod. Phys. 83, 33 (2011).

[3] L.-M. Duan, M. D. Lukin, J. I. Cirac, and P. Zoller, Nature (London) 414, 413 (2001).

[4] K. S. Choi, a. Goban, S. B. Papp, S. J. van Enk, and H. J. Kimble, Nature (London) 468, 412 (2010).

[5] Y. O. Dudin, A. G. Radnaev, R. Zhao, J.Z. Blumoff, T. A. B. Kennedy, and A. Kuzmich, Phys. Rev. Lett. 105, 260502 (2010).

[6] P. M. Ledingham, W. R. Naylor, J. J. Longdell, S. E. Beavan, and M. J. Sellars, Phys. Rev. A 81, 012301 (2010).

[7] There are also proposals to augment the DLCZ protocol with photon-echo techniques to enable temporally multimode photon-pair generation ([8,9]).

[8] C. Simon, H. de Riedmatten, and M. Afzelius, Phys. Rev. A 82, 010304(R) (2010).

[9] P. Sekatski, N. Sangouard, N. Gisin, H. de Riedmatten, and M. Afzelius, Phys. Rev. A 83, 053840 (2011).

[10] E. Fraval, M. J. Sellars, and J. J. Longdell, Phys. Rev. Lett. 92, 077601 (2004).

[11] S. E. Beavan, E. Fraval, M. J. Sellars, and J. J. Longdell, Phys. Rev. A 80, 032308 (2009).

[12] A. L. Alexander, J. J. Longdell, and M. J. Sellars, J. Opt. Soc. Am. B 24, 2479 (2007).
[13] M. P. Hedges, J. J. Longdell, Y. Li, and M. J. Sellars, Nature (London) 465, 1052 (2010).

[14] M. Afzelius, I. Usmani, A. Amari, B. Lauritzen, A. Walther, C. Simon, N. Sangouard, J. Minar, H. de Riedmatten, N. Gisin, and S. Kröll, Phys. Rev. Lett. 104, 040503 (2010).

[15] C. Clausen, I. Usmani, F. Bussi ‘eres, N. Sangouard, M. Afzelius, H. de Riedmatten, and N. Gisin, Nature (London) 469, 508 (2011).

[16] E. Saglamyurek, N. Sinclair, J. Jin, J. a. Slater, D. Oblak, F. Bussières, M. George, R. Ricken, W. Sohler, and W. Tittel, Nature (London) 469, 512 (2011).

[17] Technically this is amplified spontaneous emission, however the amplification can be $\ll 1$ such that multiplephoton events are highly improbable.

[18] N. A. Kurnit, I. D. Abella, and S. R. Hartmann, Phys. Rev. Lett. 13, 567 (1964).

[19] S. E. Beavan, P. M. Ledingham, J. J. Longdell, and M. J. Sellars, Opt. Lett. 36, 1272 (2011).

[20] R. W. Equall, R. L. Cone, and R. M. Macfarlane, Phys. Rev. B 52, 3963 (1995).

[21] M. Nilsson, L. Rippe, S. Kröll, R. Klieber, and D. Suter, Phys. Rev. B 70, 214116 (2004).

[22] See Supplemental Material at http://link.aps.org/ supplemental/10.1103/PhysRevLett.109.093603 for experimental details, specifically the hole-burning method for the ensemble preparation, and the absorptive filter preparation. Also included is an explanation of how the autocorrelation measurement is performed with a single APD.

[23] L. Mandel and E. Wolf, Optical Coherence and Quantum Optics (Cambridge University Press, Cambridge, England, 1995).

[24] P. M. Ledingham, W. R. Naylor, and J. J. Longdell , preceding Letter, Phys. Rev. Lett. 109, LS12972 (2012). 